\title{
Luteolin inhibits SH-SY5Y cell apoptosis through suppression of the nuclear transcription factor- $\kappa B$, mitogen-activated protein kinase and protein kinase B pathways in lipopolysaccharide-stimulated cocultured BV2 cells
}

\author{
LIHONG ZHU $^{1 *}$, WEI BI ${ }^{2 *}$, DAN LU ${ }^{1}$, CHANJUAN ZHANG $^{1}$, XIAOMING SHU $^{1}$ and DAXIANG LU ${ }^{1}$ \\ ${ }^{1}$ Department of Pathophysiology, Institute of Brain Research, School of Medicine, Jinan University, Guangzhou, \\ Guangdong 510632; ${ }^{2}$ Department of Neurology, First Affiliated Hospital of Jinan University, \\ Guangzhou, Guangdong 510630, P.R. China
}

Received August 12, 2013; Accepted January 6, 2014

DOI: $10.3892 /$ etm.2014.1564

\begin{abstract}
Microglial activation is one of the causative factors for neuroinflammation, which is associated with the pathophysiology of neurodegenerative diseases. Our previous study showed that the flavonoid luteolin inhibited several pro-inflammatory enzymes and pro-inflammatory cytokines that are induced by activated microglia; however, its effect on signaling pathways is currently unknown. The present study examined the effects of luteolin on signaling pathways stimulated by lipopolysaccharide (LPS), including Toll-like receptor-4 (TLR-4), nuclear transcription factor- $\kappa \mathrm{B}(\mathrm{NF}-\kappa \mathrm{B})$, mitogen-activated protein kinase (MAPK) family and protein kinase B (Akt) pathways in murine microglial BV2 cells. In addition, BV2 microglia and SH-SY5Y neuroblastoma cells were cocultured to observe the indirect neuroprotective effects of luteolin. Luteolin inhibited the LPS-stimulated expression of TLR-4. In addition, luteolin blocked LPS-induced NF- $\mathrm{B}$, p38, JNK and Akt activation, but had no effect on ERK. When SH-SY5Y cells were cocultured with LPS-stimulated BV2 microglia, pretreatment with luteolin increased neuronal viability and reduced the number of apoptotic cells. These data suggest that luteolin has a beneficial effect on neuroinflammatory events in neurodegenerative diseases via suppression of the NF- $\mathrm{B}, \mathrm{MAPK}$ and Akt pathways in activated microglial cells.
\end{abstract}

Correspondence to: Dr Daxiang Lu, Department of Pathophysiology, School of Medicine, Jinan University, 601 Huangpu Road West, Guangzhou, Guangdong 510632, P.R. China

E-mail: ldx_jnu@sina.com

*Contributed equally

Key words: protein kinase $\mathrm{B}$, luteolin, mitogen-activated protein kinase, microglia, neuroprotection, nuclear transcription factor $\kappa \mathrm{B}$, Toll-like receptor 4, neuroinflammation

\section{Introduction}

Microglia are the cells responsible for innate immunity in the central nervous system and accumulating evidence suggests that activated microglia modulate the development and/or progression of Alzheimer's disease, Parkinson's disease and amyotrophic lateral sclerosis, among other diseases (1-3). Exposure to lipopolysaccharide (LPS), $\beta$-amyloid or interferon (IFN)- $\gamma$ activates microglia, inducing the secretion of a variety of pro-inflammatory mediators and potentially neurotoxic compounds (4,5). Receptor binding of cytokines stimulates a variety of intracellular signaling pathways that have been implicated in neurodegenerative disorders, including activation of nuclear transcription factor- $\kappa \mathrm{B}(\mathrm{NF}-\kappa \mathrm{B})$, the mitogen-activated protein kinase (MAPK) family and protein kinase $B$ (Akt). These are the most important molecules that control the synthesis and release of pro-inflammatory substances from activated microglia (6-8). Epidemiological studies suggest that inhibition of microglial activation attenuates the severity of neurotoxicity $(9,10)$. The inhibition of activated microglia is therefore an important therapeutic target for neurodegenerative disorders.

Luteolin (3',4',5,7-tetrahydroxyflavone), a flavone that has been identified in celery, green pepper, perilla leaves and seeds, and at high concentrations in chamomile, exhibits strong anti-inflammatory, antioxidant and free-radical scavenging properties. In addition, luteolin has been shown to inhibit the LPS-induced production of tumor necrosis factor alpha (TNF- $\alpha$ ) and nitric oxide (NO) in an activated macrophage-like cell line (11). Luteolin also reduces the production of LPS-induced pro-inflammatory cytokines in intestinal epithelial cells, mouse bone marrow-derived dendritic cells (12), rat fibroblasts (13) and human gingival fibroblasts (14). Furthermore, in a previous study we observed that luteolin inhibited the secretion of several proinflammatory enzymes and pro-inflammatory cytokines by activated microglia (15). However, the mechanism by which luteolin inhibits microglial inflammation is not completely understood. Much less is known about the role of luteolin in 
neuroprotection and regulation of the underlying signaling pathways.

In the present study, the effects of luteolin on Toll-like receptor-4 (TLR-4) expression and the NF- $\kappa \mathrm{B}$, MAPK and Akt signaling pathways were investigated using LPS-stimulated BV2 cells, a murine microglial cell line. In further experiments using a microglial-neuronal coculture system, the protective effects of luteolin against microglial-mediated LPS neurotoxicity, and therefore its potential role in the prevention of neurodegenerative diseases were investigated.

\section{Materials and methods}

Materials. Luteolin (purity > 98\%; molecular weight, 286.24; chemical formula $\mathrm{C}_{15} \mathrm{H}_{10} \mathrm{O}_{6}$ ), LPS, dimethylsulfoxide (DMSO) and 3-(4,5-dimethylthiazol-2-yl)-2,5-diphenyltetrazolium bromide (MTT) were purchased from Sigma (St. Louis, MO, USA). Antibodies against NF- $\kappa \mathrm{B}$ p65, p38, phosphorylated p38 (p-p38), JNK, phosphorylated JNK (p-JNK), ERK, phosphorylated ERK (p-ERK), Akt and phosphorylated Akt (p-Akt) were obtained from Cell Signaling Technology (Beverly, MA, USA). Antibodies against TLR-4 were obtained from Santa Cruz Biotechnology (Santa Cruz, CA, USA). Mouse anti- $\beta$-actin antibody was purchased from Sigma.

Cell culture. BV2 immortalized murine microglia were provided by the Cell Culture Center of the Chinese Academy of Medical Sciences (Beijing, China). The human neuroblastoma cell line SH-SY5Y was donated by Dr Enxiang Tao. The cells were cultured in Dulbecco's modified Eagle's medium (DMEM) supplemented with 10\% fetal bovine serum (FBS), $100 \mathrm{U} / \mathrm{ml}$ penicillin and $100 \mu \mathrm{g} / \mathrm{ml}$ streptomycin in a humidified atmosphere of $5 \% \mathrm{CO}_{2}$ at $37^{\circ} \mathrm{C}$. In all experiments, the $\mathrm{BV} 2$ microglia were pretreated with the indicated concentrations of luteolin for $1 \mathrm{~h}$ prior to the addition of LPS $(1.0 \mu \mathrm{g} / \mathrm{ml})$ in serum-free DMEM.

Immunofluorescence staining. For immunofluorescence staining, the cells were fixed with $4 \%$ paraformaldehyde for $15 \mathrm{~min}$, permeabilized with $0.1 \%$ Triton X-100 for $10 \mathrm{~min}$ and blocked with $5 \%$ bovine serum albumin (BSA) for $30 \mathrm{~min}$. The cells were then incubated with primary antibody to NF- $\kappa \mathrm{B}$ p65 (1:100 dilution) overnight at $4^{\circ} \mathrm{C}$. After washing three times with PBS, the cells were incubated with secondary antibody conjugated to rhodamine for $1 \mathrm{~h}$ (Cell Signaling Technology). The nuclei were stained with Hoechst 33258. Fluorescent images were captured using a laser scanning confocal microscope (LSM 510 META; Carl Zeiss, Stuttgart, Germany).

Total RNA isolation and quantitative PCR (qPCR) analysis. Total RNA was isolated with TRIzol reagent (Invitrogen Life Technologies, Carlsbad, CA, USA) according to the manufacturer's instructions. Total RNA $(1.0 \mu \mathrm{g})$ was reverse transcribed using M-MLV reverse transcriptase (Promega, Madison, WI, USA) to produce cDNA. The primers used for qPCR were as follows: $T L R-4,5$ '-GCT TTC ACC TCT GCC TTC AC-3' and 5'-CCA ACG GCT CTG AAT AAA GTG-3'; and GAPDH, 5'-TCA CCA CCA TGG AGA AGG C-3' and 5'-GCT AAG CAG TTG GTG GTG CA-3'. The following qPCR conditions were used: 40 cycles of denaturation at $94^{\circ} \mathrm{C}$ for $20 \mathrm{sec}$, annealing at $62^{\circ} \mathrm{C}$ for $30 \mathrm{sec}$ and extension at $72^{\circ} \mathrm{C}$ for $30 \mathrm{sec}$. SYBR Green qPCR Master mix 2 (Takara Bio, Inc., Shiga, Japan) was used in all samples and the reactions were carried out in a $20-\mu 1$ reaction volume using a LightCycler LC480 qPCR instrument (Roche Diagnostics, Basel, Switzerland). The mRNA expression levels of target genes relative to glyceraldehyde-3-phosphate dehydrogenase (GAPDH, a housekeeping gene used as an endogenous control) were calculated according to the standard curves.

Western blot analysis. BV2 microglia were harvested and lysed in RIPA buffer [1 mM ethylenediaminetetraacetic acid (EDTA), $150 \mathrm{mM} \mathrm{NaCl}, 1 \%$ igepal (CA-630), 0.1\% sodium dodecyl sulfate (SDS), $0.5 \%$ sodium deoxycholate and $50 \mathrm{mM}$ Tris $\mathrm{HCl}$; $\mathrm{pH} 8.0$ (Sigma)]. Equal amounts of protein were separated by $8-12 \%$ sodium dodecyl sulfate polyacrylamide gel electrophoresis (SDS-PAGE), transferred to polyvinylidene fluoride (PVDF) membranes, blocked with 5\% nonfat milk for $2 \mathrm{~h}$ and incubated with primary antibodies at $4{ }^{\circ} \mathrm{C}$ overnight. Following incubation with appropriate secondary antibodies conjugated to horseradish peroxidase (goat anti-rabbit secondary antibody was obtained from Cell Signaling Technology), immunoblots were exposed on film using electrochemiluminescence (ECL) western detection reagent (Amersham Pharmacia Biotech, Amersham, UK). The bands were quantified by the optical density ratio using $\beta$-actin as a control.

Cytotoxicity assay in a coculture of microglia and neurons. SH-SY5Y cells were grown in the bottom of wells, BV2 cells were then grown in culture inserts (pore size $0.4 \mu \mathrm{m}$; Corning, New York, NY, USA) and $1.0 \mu \mathrm{g} / \mathrm{ml}$ LPS was added to the culture insert. In this coculture system, the microglia were able to communicate with the neurons through a semipermeable membrane, which avoids direct contact between the two cellular systems (16). After coculture for $24 \mathrm{~h}$, the SH-SY5Y cells were incubated with MTT solution $(0.5 \mathrm{mg} / \mathrm{ml}$ in PBS) for $4 \mathrm{~h}$ at $37^{\circ} \mathrm{C}$. The culture supernatants were then removed, the resulting formazan crystals were dissolved in DMSO and the absorbance was read at $570 \mathrm{~nm}$ with a microplate reader (ReTiSoft Inc., Mannedorf, Switzerland). Cell survival was expressed as the ratio of absorbance (percentage survival) compared with a DMSO control.

Detection of apoptosis in a coculture of microglia and neurons. In the coculture system described above, apoptotic SH-SY5Y cells were detected by the terminal deoxynucleotidyl transferase-mediated dUTP nick-end labeling (TUNEL) assay. Following each treatment, the TUNEL assay was performed according to manufacturer's instructions (Roche Diagnostics Corporation, Indianapolis, IN, USA) and all nuclei were counterstained with $5 \mathrm{mg} / \mathrm{ml}$ Hoechst 33342 for $10 \mathrm{~min}$ at $37^{\circ} \mathrm{C}$. The labeled SH-SY5Y cells were examined with a laser scanning confocal microscope. SH-SY5Y cells were considered to be apoptotic when their nuclei were costained with Hoechst 33342 and TUNEL. The number of apoptotic cells was counted among 100 randomly chosen neurons observed on several optic fields.

Statistical analysis. Quantitative data are presented as the mean \pm standard error of the mean (SEM) of at least three 


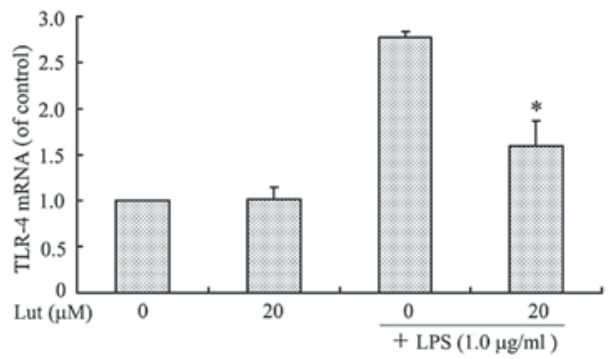

Figure 1. Effects of luteolin on the expression of TLR-4 in LPS-stimulated BV2 microglia. qPCR analysis of TLR-4 mRNA expression. Cells were treated with $20 \mu \mathrm{M}$ luteolin for $1 \mathrm{~h}$ prior to the addition of LPS $(1.0 \mu \mathrm{g} / \mathrm{ml})$ for $1 \mathrm{~h}$. Total RNA was then isolated and analyzed for TLR-4 mRNA expression using qPCR. TLR-4 mRNA expression levels were calculated relative to a GAPDH control using standard curves. Data were collected from three independent experiments each carried out in triplicate. "Indicates a significant difference $(\mathrm{P}<0.05)$ relative to cells treated with LPS in the absence of luteolin. TLR-4, Toll-like receptor-4; LPS, lipopolysaccharide; GAPDH, glyceraldehyde-3-phosphate dehydrogenase; Lut, luteolin.

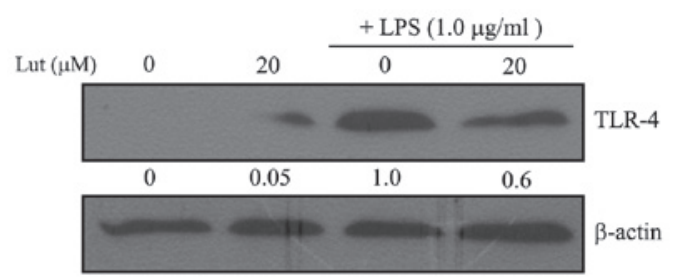

Figure 2. Effects of luteolin on the expression of TLR-4 in LPS-stimulated BV2 microglia. Western blot analysis of TLR-4. Cells were incubated with $20 \mu \mathrm{M}$ luteolin for $1 \mathrm{~h}$ prior to incubation with LPS $(1.0 \mu \mathrm{g} / \mathrm{ml})$ for $24 \mathrm{~h}$. Cell lysates were then prepared and subjected to western blotting. TLR-4 protein expression was quantified by OD ratio using $\beta$-actin as a control. Data were collected from three independent experiments each carried out in triplicate. "Indicates a significant difference $(\mathrm{P}<0.05)$ relative to cells treated with LPS in the absence of luteolin. TLR-4, Toll-like receptor-4; LPS, lipopolysaccharide; OD, optical density; Lut, luteolin.

independent experiments. Comparisons between two groups were analyzed using the Student's t-test. $\mathrm{P}<0.05$ was considered to indicate a statistically significant result.

\section{Results}

Luteolin suppresses TLR-4 expression in LPS-stimulated BV2 microglia. To examine the effect of luteolin on TLR-4 expression, the levels of TLR-4 mRNA and protein in LPS-stimulated BV2 microglia were measured. The BV2 microglia were pretreated with luteolin for $1 \mathrm{~h}$ and then stimulated with LPS for $1 \mathrm{~h}$ prior to $\mathrm{qPCR}$ or for $24 \mathrm{~h}$ prior to western blotting. As shown in Figs. 1 and 2, TLR-4 mRNA and protein levels increased in LPS-stimulated BV2 microglia, but both were markedly suppressed by treatment with luteolin. This result indicates that luteolin suppressed TLR-4 expression and therefore may lead to inhibition of activation of the NF- $\mathrm{NB}, \mathrm{MAPK}$ and Akt pathways.

Effects of luteolin on the $N F-\kappa B$ signaling pathway. Activation of $\mathrm{NF}-\kappa \mathrm{B}$ leads to its translocation to the nucleus where it mediates the transcriptional regulation of pro-inflammatory genes. The activation and nuclear translation of $\mathrm{NF}-\kappa \mathrm{B}$ is a key step in LPS-stimulated microglial activation. The regulation
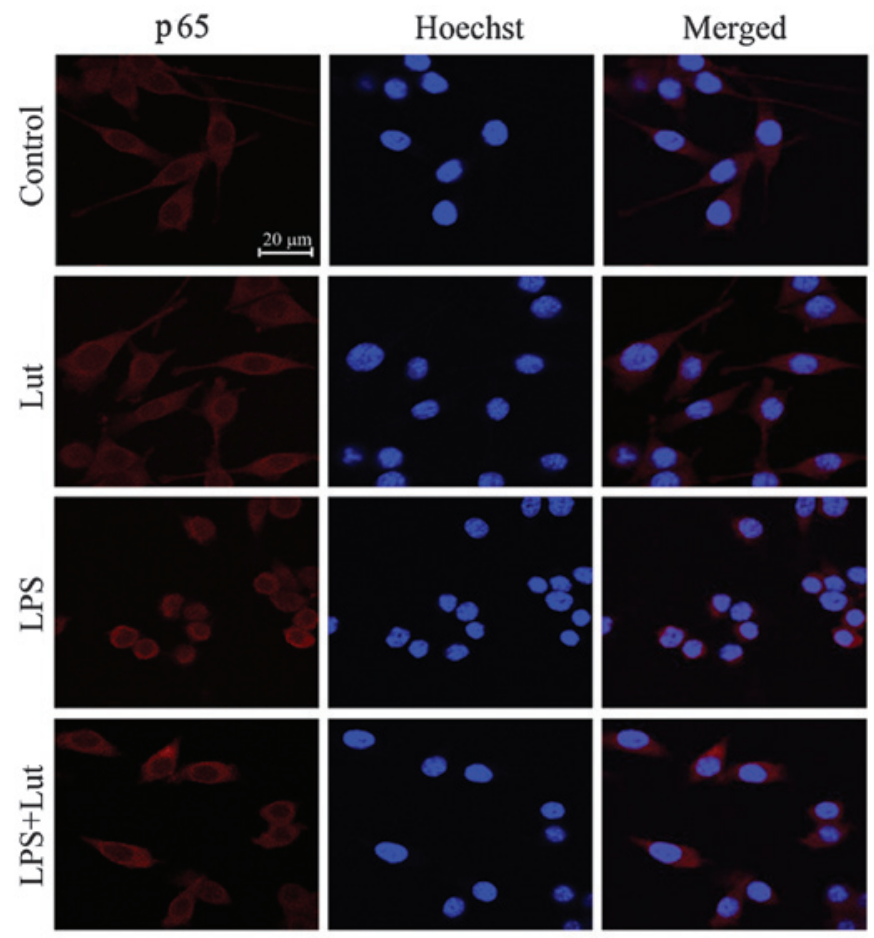

Figure 3. Downregulation of NF- $\mathrm{B}$ activation by luteolin in LPS-stimulated BV2 microglia. Immunofluorescent staining showing the cellular distribution of the NF-кB p65 subunit (red). Cells were pretreated with luteolin for $1 \mathrm{~h}$, followed by LPS $(1.0 \mu \mathrm{g} / \mathrm{ml})$ stimulation for $1 \mathrm{~h}$. Hoechst 33258 (blue) was used to visualize the nuclei. Data was collected from three independent experiments each carried out in triplicate. Scale bar represents $20 \mu \mathrm{m}$. LPS, lipopolysaccharide; NK- $\kappa \mathrm{B}$, nuclear transcription factor $\kappa \mathrm{B}$; Lut, luteolin.

of NF- $\kappa \mathrm{B}$ by luteolin was investigated using immunofluorescence staining. As shown in Fig. 3, the NF- $\kappa$ B p65 subunit was primarily retained in the cytoplasm in unstimulated cells; however, following stimulation with LPS, cytoplasmic NF- $\mathrm{B}$ p65 levels were reduced, with a corresponding increase in nuclear NF- $\kappa \mathrm{B}$ p 65 . Treatment with $20 \mu \mathrm{M}$ luteolin significantly blocked the activation of $\mathrm{NF}-\kappa \mathrm{B}$ p65 nuclear translocation in LPS-stimulated BV-2 cells. This result suggests that luteolin suppresses pro-inflammatory enzymes and pro-inflammatory cytokines by inhibiting NF- $\kappa \mathrm{B}$ activation.

Effects of luteolin on the MAPK signaling pathway. To investigate whether the inhibition of $\mathrm{NF}-\kappa \mathrm{B}$ activation by luteolin is mediated via the MAPK pathway, the phosphorylation of three MAPK molecules, p38 MAPK, JNK and ERK1/2, was examined in LPS-stimulated BV-2 cells. As shown in Fig. 4, LPS rapidly activated MAPKs within $15 \mathrm{~min}$ of LPS stimulation, while luteolin at $20 \mu \mathrm{M}$ markedly inhibited the LPS-induced phosphorylation of p38 and JNK, but had no effect on ERK phosphorylation. The levels of non-phosphorylated p38, JNK and ERK were unaffected by LPS or luteolin treatment.

Effects of luteolin on the Akt signaling pathway. The effect of luteolin on Akt was then examined. As shown in Fig. 5, luteolin significantly inhibited the LPS-induced activation of Akt. The results suggest that inhibition of Akt by luteolin may contribute to the suppression of LPS-induced NF- $\kappa \mathrm{B}$ activation and the expression of inflammatory mediators in BV2 cells. 


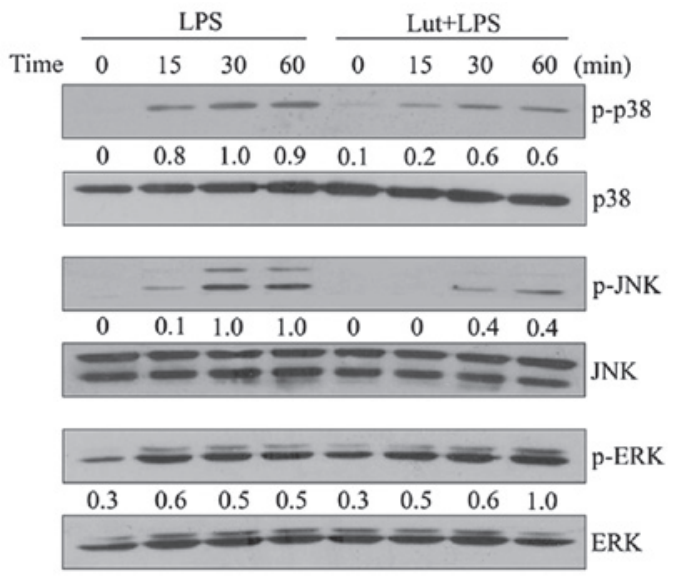

Figure 4. Downregulation of MAPK phosphorylation by luteolin in LPS-stimulated BV2 microglia. BV2 microglial cells were treated with LPS $(1.0 \mu \mathrm{g} / \mathrm{ml})$ for the indicated time with or without pretreatment with $20 \mu \mathrm{M}$ luteolin for $1 \mathrm{~h}$. Cell lysates were prepared and used for western blot analysis with antibodies against the indicated MAPKs. Expression levels of phosphorylated MAPKs were quantified by OD ratios relative to controls, and these data are shown between the relevant blots. LPS rapidly activated MAPKs including p38, JNK, and ERK within 15 min of LPS stimulation, while pretreatment of cells with $20 \mu \mathrm{M}$ luteolin markedly inhibited the LPS-induced phosphorylation of p38 and JNK. Data were collected from three independent experiments each carried out in triplicate. MAPK, mitogen-activated protein kinase; LPS, lipopolysaccharide; OD, optical density.

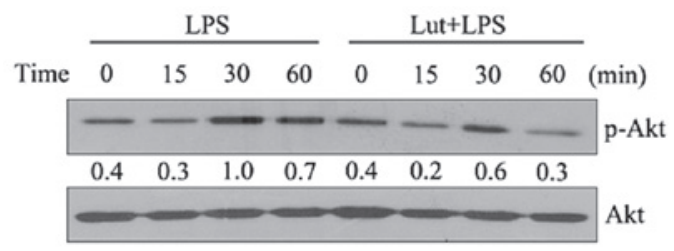

Figure 5. Downregulation of Akt phosphorylation by luteolin in LPS-stimulated BV2 microglia. The BV2 microglial cells were treated with LPS $(1.0 \mu \mathrm{g} / \mathrm{ml})$ for the indicated time with or without pretreatment with $20 \mu \mathrm{M}$ luteolin for $1 \mathrm{~h}$. Cell lysates were prepared and used for western blot analysis with antibodies against Akt and p-Akt. Expression levels of p-Akt were quantified by OD ratios relative to control. LPS treatment induced Akt phosphorylation, this process was significantly suppressed by luteolin. Data were collected from three independent experiments each carried out in triplicate. Akt, protein kinase B; LPS, lipopolysaccharide; p-Akt, phosphorylated protein kinase B; Lut, luteolin; OD, optical density.

Luteolin decreases microglial-induced SH-SY5Y cell death in a coculture system. In order to investigate whether luteolin protects against the neuronal death induced by microglial activation, a coculture system with SH-SY5Y neuronal cells and BV2 microglia was used. LPS at concentrations of 0.1, 1.0 or $10.0 \mu \mathrm{g} / \mathrm{ml}$ were not observed to induce cell death in the SH-SY5Y cells (data not shown). Next, the SH-SY5Y cell viability following coculture with LPS-activated BV2 microglia was examined using the MTT assay. As shown in Fig. 6, SH-SY5Y cells in control inserts in the absence of LPS-stimulated BV2 microglia did not undergo cell death. By contrast, LPS treatment alone led to a high level of SH-SY5Y cell death in the coculture, suggesting that the LPS-activated microglia secreted pro-inflammatory cytokines that were able to migrate through the insert, inducing the death of the neuronal cells. Treatment with luteolin markedly reduced

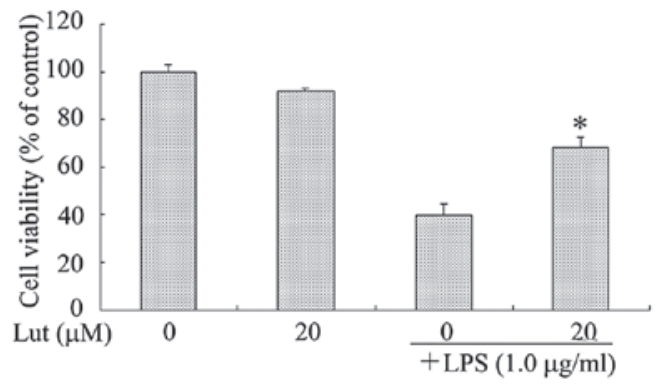

Figure 6. Effect of luteolin on SH-SY5Y cell survival in an LPS-induced microglial-neuronal coculture system. SH-SY5Y cells were cocultured with LPS-activated BV2 microglia with or without pretreatment with $20 \mu \mathrm{M}$ luteolin for $24 \mathrm{~h}$. An MTT assay was used to determine SH-SY5Y cell viability. ${ }^{*} \mathrm{P}<0.05$ compared with the LPS group. Lut, luteolin; LPS, lipopolysaccharide; MTT, 3-(4,5-dimethylthiazol-2-yl)-2,5-diphenyltetrazolium bromide.
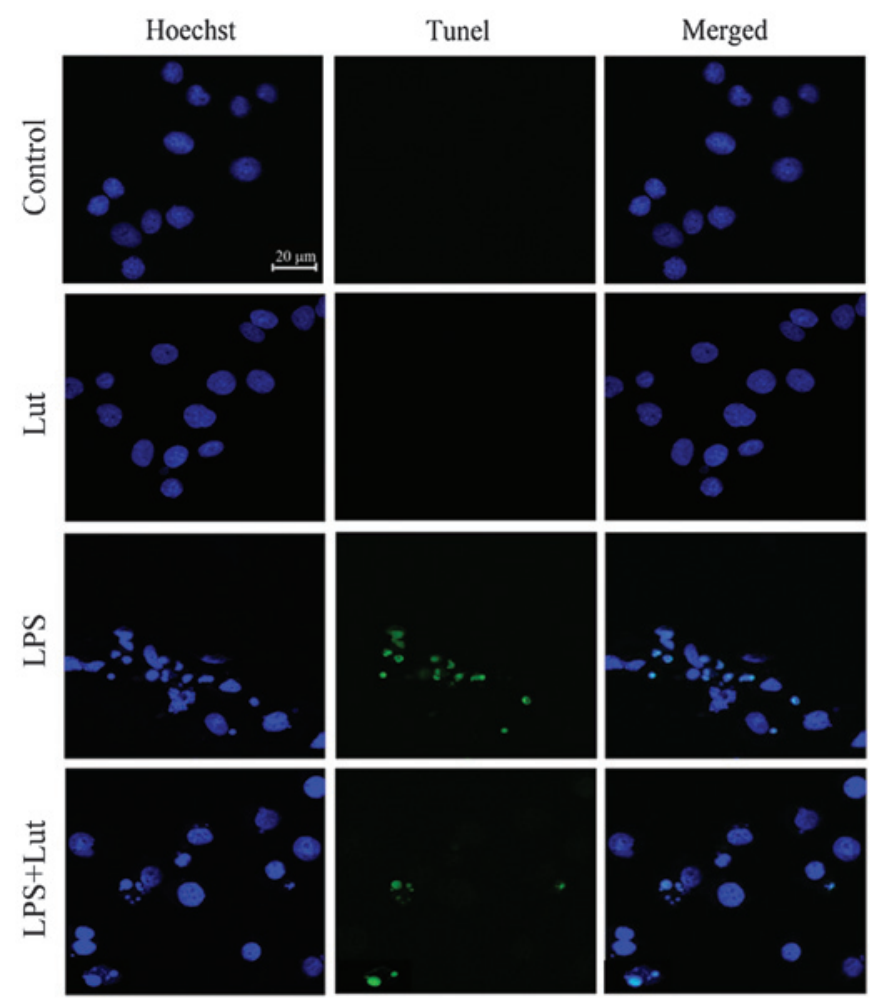

Figure 7. Effect of luteolin on SH-SY5Y cell survival in an LPS-induced microglial-neuronal coculture system. SH-SY5Y cells were cocultured with LPS-activated BV2 microglia with or without pretreatment with $20 \mu \mathrm{M}$ luteolin for $24 \mathrm{~h}$. Immunofluorescence detection of apoptotic SH-SY5Y cells cocultured with BV2 microglia. Scale bar represents $20 \mu \mathrm{m}$. Lut, luteolin; LPS, lipopolysaccharide.

the death of the SH-SY5Y cells; cell viability was increased by $\sim 28.6 \%$ when the LPS-stimulated BV2 microglia were pretreated with luteolin (Fig. 6).

Apoptosis was determined by the TUNEL assay. As shown in Fig. 7, SH-SY5Y nuclei were stained with Hoechst 33342 (blue) and apoptotic neurons were stained using the TUNEL technique (green). Coculture with BV2 microglia exposed to LPS alone resulted in a significant increase in the number of apoptotic SH-SY5Y cells compared with the number of untreated cells. The administration of luteolin reduced the 


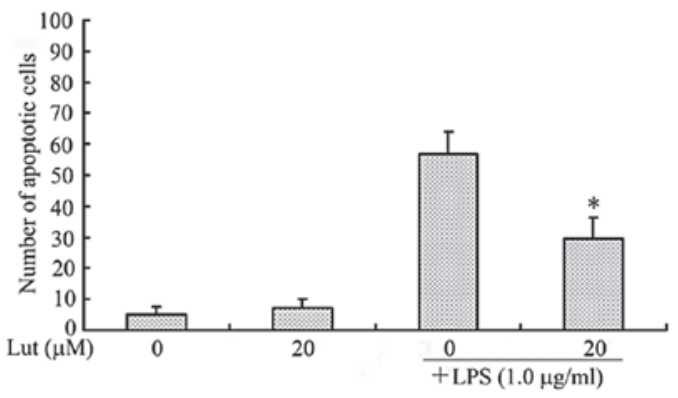

Figure 8. Effect of luteolin on SH-SY5Y cell survival in an LPS-induced microglial-neuronal coculture system. SH-SY5Y cells were cocultured with LPS-activated BV2 microglia with or without pretreatment with $20 \mu \mathrm{M}$ luteolin for $24 \mathrm{~h}$. The number of apoptotic neurons counted on double-stained slides (merged) from a total of 100 nuclei. Data were collected from three independent experiments each carried out in triplicate. "Indicates a significant difference $(\mathrm{P}<0.05)$ relative to cells treated with LPS in the absence of luteolin. Lut, luteolin; LPS, lipopolysaccharide.

number of apoptotic SH-SY5Y cells (Fig. 8). These results clearly demonstrate that luteolin protected neurons from microglial-mediated LPS neurotoxicity, supporting its potential role in the prevention of neurodegenerative diseases.

\section{Discussion}

The flavonoid luteolin has been shown to inhibit LPS-induced IL-6 production in the brain by inhibiting the JNK signaling pathway and the activation of AP-1 in microglia (17). Luteolin also suppressed microglial TNF- $\alpha$ and IL- 6 production stimulated by IFN- $\gamma$ in the presence of CD40 ligation, and markedly inhibited the IFN- $\gamma$-induced phosphorylation of STAT1 (18). These data suggest that this flavonoid is a potent modulator of microglial activation and affects several signaling pathways, leading to a unique phenotype with anti-inflammatory, anti-oxidative and neuroprotective characteristics (19). Previous findings suggest dietary luteolin enhances spatial working memory by mitigating microglial-associated inflammation in the hippocampus (20). Our previous observations confirm the inhibitory effects of luteolin on pro-inflammatory cytokine expression in microglia (15). However, the mechanism by which luteolin mediates these anti-inflammatory effects on microglia is not completely understood and few studies have explored the impact of luteolin on neuroprotection.

In vitro, microglia may be activated experimentally with the bacterial cell wall component LPS. Internalization of TLR-4, rendering microglial cells less sensitive to activation by LPS, plays a role in inducing a reduction in TNF- $\alpha$ production by BV-2 microglial cells (21). TLR-4 is a member of the TLR family of pattern recognition receptors that generate innate immune responses to pathogens by activating a cascade of pro-inflammatory events (22). Therefore, treatments that attenuate TLR-4-associated inflammatory cascades may prove beneficial in ameliorating microglial activation and preventing neurodegenerative processes. The results of the present study indicate that luteolin pretreatment inhibited the upregulation of TLR-4 expression induced by LPS at the transcriptional and translational levels. It was therefore hypothesized that the underlying molecular mechanisms involved include interference with the LPS-triggered increase in TLR-4 expression.
The results of the present study indicate that luteolin may inhibit NF- $\mathrm{B}$, p38, JNK, MAPK and Akt activation through the suppression of TLR-4 expression.

Activation of $N F-\kappa B$ leads to its translocation to the nucleus where it mediates the transcriptional regulation of pro-inflammatory genes (23). The activation and nuclear translation of $\mathrm{NF}-\kappa \mathrm{B}$ is a key step in LPS-stimulated microglial activation (24). In the current study, treatment with $20 \mu \mathrm{M}$ luteolin significantly blocked NF- $\mathrm{B}$ p65 nuclear translocation in LPS-stimulated BV-2 cells. In our previous study, a luciferase reporter assay was performed to investigate the possibility that luteolin inhibits NF- $\kappa \mathrm{B}$ transcriptional activity, and the possibility that luteolin blocks the phosphorylation and subsequent degradation of I $\mathrm{B}$ in LPS-induced BV2 cells was investigated; it was observed that luteolin causes a marked inhibition of $N F-\kappa B$ p65 nuclear translocation (15). These results, also confirmed by the current study, suggest that luteolin suppresses pro-inflammatory enzymes and pro-inflammatory cytokines through the inhibition of $\mathrm{NF}-\kappa \mathrm{B}$ activation.

There is evidence that MAPKs play a key role in the regulation of the synthesis and release of pro-inflammatory substances by activated microglia (25). LPS is known to activate various MAPKs, including p38, JNK and ERK. The MAPKs tested in this study (p38, JNK and ERK1/2) were activated in glia and neurons following LPS treatment, suggesting their involvement in glial activation and the neuronal response to diffusible, glia-derived neurotoxic molecules (26). To investigate whether the inhibition of $N F-\kappa B$ activation by luteolin is mediated via the MAPK pathway, the phosphorylation of three MAPK molecules, p38 MAPK, JNK and ERK1/2 in LPS-stimulated BV-2 cells was examined. LPS rapidly activated MAPKs within 15 min of LPS stimulation, while luteolin at $20 \mu \mathrm{M}$ markedly inhibited the LPS-induced phosphorylation of p38 and JNK, but had no effect on ERK. However, further studies are necessary to support this conclusion.

Multiple signaling pathways, such as those involving MAPKs and Akt, are involved in LPS-stimulated signal transduction and lead to the activation of $N F-\kappa B$ and the subsequent induction of pro-inflammatory gene expression $(27,28)$. Akt is activated via the phosphoinositide-3-OH kinase (PI3K) pathway, an important pathway regulating inflammation and immunity (29). The results of the present study indicate that luteolin also significantly inhibited the LPS-induced activation of Akt. The data suggest that inhibition of Akt by luteolin may contribute to the suppression of LPS-induced NF- $\kappa \mathrm{B}$ activation and the expression of inflammatory mediators in BV2 cells.

Neurotoxic microglial-neuronal interactions have been implicated in the pathogenesis of various neurodegenerative diseases (30). Microglial activation has been shown to promote the production of inflammatory cytokines leading to neuronal apoptosis $(31,32)$. In order to investigate whether luteolin is able to rescue neurons from death induced by microglial activation, SH-SY5Y cells and BV2 microglia in a coculture system were used in the present study. The results clearly indicate that when SH-SY5Y cells were cocultured with LPS-stimulated BV2 microglia, neuronal cell death increased by $60.2 \%$ and the number of apoptotic neurons increased by $57.0 \%$. However, treatment with luteolin in this LPS-induced coculture system increased cell viability by $28.6 \%$ and reduced the apoptotic cell number by $27.0 \%$. These data suggest that 
luteolin inhibited SH-SY5Y cell apoptosis via inhibition of microglial activation in the microglial-neuronal coculture system. These results provide strong evidence that luteolin protects neurons from microglial-mediated LPS neurotoxicity. However, further in vivo investigation of this activity is necessary in order to clarify the molecular mechanisms involved and assess the full medicinal potential of luteolin.

In conclusion, the present study demonstrated that luteolin inhibited the LPS-stimulated expression of TLR-4. Luteolin also blocked LPS-induced NF- $\mathrm{B}$, p38, JNK and Akt activation, but had no effect on ERK. When SH-SY5Y cells were cocultured with LPS-stimulated BV2 microglia, pretreatment with luteolin increased neuronal viability and reduced the number of apoptotic cells. These observations suggest that luteolin has a therapeutic application in the treatment of neurodegenerative diseases.

\section{Acknowledgements}

This study was supported by grants from the National Natural Science Foundation of China (Nos. 81102449 and 81200930), the Natural Science Foundation of Guangdong Province (Nos. S2011040003038 and S2012040007768) and the Fundamental Research Funds for the Central Universities (No. 21612423).

\section{References}

1. Bernardi A, Frozza RL, Meneghetti A, Hoppe JB Battastini AM, Pohlmann AR, Guterres SS and Salbego CG: Indomethacin-loaded lipid-core nanocapsules reduce the damage triggered by A $\beta 1-42$ in Alzheimer's disease models. Int J Nanomedicine 7: 4927-4942, 2012.

2. Yokoyama H, Uchida H, Kuroiwa H, Kasahara J and Araki T: Role of glial cells in neurotoxin-induced animal models of Parkinson's disease. Neurol Sci 32: 1-7, 2011.

3. Dibaj P, Zschüntzsch J, Steffens H, Scheffel J, Göricke B, Weishaupt JH, Le Meur K, Kirchhoff F, Hanisch UK, Schomburg ED and Neusch C: Influence of methylene blue on microglia-induced inflammation and motor neuron degeneration in the SOD1(G93A) model for ALS. PLoS One 7: e43963, 2012.

4. McGeer EG and McGeer PL: Neuroinflammation in Alzheimer's disease and mild cognitive impairment: a field in its infancy. J Alzheimers Dis 19: 355-361, 2010

5. Mrak RE and Griffin WS: Glia and their cytokines in progression of neurodegeneration. Neurobiol Aging 26: 349-354, 2005.

6. Anisman H: Cascading effects of stressors and inflammatory immune system activation: implications for major depressive disorder. J Psychiatry Neurosci 34: 4-20, 2009.

7. Ha SK, Lee P, Park JA, Oh HR, Lee SY, Park JH, et al: Apigenin inhibits the production of NO and PGE2 in microglia and inhibits neuronal cell death in a middle cerebral artery occlusion-induced focal ischemia mice model. Neurochem Int 52: 878-886, 2008.

8. Lyons A, Downer EJ, Crotty S, Nolan YM, Mills KH and Lynch MA: CD 200 ligand receptor interaction modulates microglial activation in vivo and in vitro: a role for IL-4. J Neurosci 27: 8309-8313, 2007.

9. Qian L, Flood PM and Hong JS: Neuroinflammation is a key player in Parkinson's disease and a prime target for therapy. J Neural Transm 117: 971-979, 2010.

10. Krause DL and Müller N: Neuroinflammation, microglia and implications for anti-inflammatory treatment in Alzheimer's disease. Int J Alzheimers Dis 2010: 732806, 2010.

11. Park E, Kum S, Wang C, Park SY, Kim BS and Schuller-Levis G: Anti-inflammatory activity of herbal medicines: Inhibition of nitric oxide production and tumor necrosis factor-alpha secretion in an activated macrophage-like cell line. Am J Chin Med 33: 415-424, 2005
12. Kim JS and Jobin C: The flavonoid luteolin prevents lipopolysaccharide-induced NF- $\kappa \mathrm{B}$ signaling and gene expression by blocking I $\mathrm{B}$ kinase activity in intestinal epithelial cells and bone-marrow derived dendritic cells. Immunology 115: 375-387, 2005.

13. Kim SH, Shin KJ, Kim D, Kim YH, Han MS, Lee TG, et al: Luteolin inhibits the nuclear factor- $\kappa \mathrm{B}$ transcriptional activity in Rat-1 fibroblasts. Biochem Pharmacol 66: 955-963, 2003.

14. Gutiérrez-Venegas G, Kawasaki-Cárdenas P, Arroyo-Cruz SR and Maldonado-Frías S: Luteolin inhibits lipopolysaccharide actions on human gingival fibroblasts. Eur J Pharmacol 541: 95-105, 2006

15. Zhu LH, Bi W, Qi RB, Wang HD and Lu DX: Luteolin inhibits microglial inflammation and improves neuron survival against inflammation. Int J Neurosci 121: 329-336, 2011.

16. Bureau G, Longpré F and Martinoli MG: Resveratrol and quercetin, two natural polyphenols, reduce apoptotic neuronal cell death induced by neuroinflammation. J Neurosci Res 86: 403-410, 2008

17. Jang S, Kelley KW and Johnson RW: Luteolin reduces IL-6 production in microglia by inhibiting JNK phosphorylation and activation of AP-1. Proc Natl Acad Sci USA 105: 7534-7539, 2008.

18. Rezai-Zadeh K, Ehrhart J, Bai Y, Sanberg PR, Bickford P, Tan J and Shytle RD: Apigenin and luteolin modulate microglial activation via inhibition of STAT1-induced CD40 expression. J Neuroinflammation 5: 41, 2008.

19. Dirscherl K, Karlstetter M, Ebert S, Kraus D, Hlawatsch J, Walczak Y, Moehle C, Fuchshofer R and Langmann T: Luteolin triggers global changes in the microglial transcriptome leading to a unique anti-inflammatory and neuroprotective phenotype. J Neuroinflammation 7: 3, 2010.

20. Jang S, Dilger RN and Johnson RW: Luteolin inhibits microglia and alters hippocampal-dependent spatial working memory in aged mice. J Nutr 140: 1892-1898, 2010.

21. Willis LM, Bielinski DF, Fisher DR, Matthan NR and Joseph JA: Walnut extract inhibits LPS-induced activation of BV-2 microglia via internalization of TLR4: Possible involvement of phospholipase D2. Inflammation 33: 325-333, 2010.

22. Medzhitov R: Toll-like receptors and innate immunity. Nat Rev Immunol 1: 135-145, 2001.

23. Ozato K, Tsujimura $\mathrm{H}$ and Tamura T: Toll-like receptor signaling and regulation of cytokine gene expression in the immune system. Biotechniques 33 (4 Suppl): S66-S75, 2002.

24. Bi W, Jing X, Zhu L, Liang Y, Liu J, Yang L, Xiao S, Xu A, Shi Q and Tao E: Inhibition of $26 \mathrm{~S}$ protease regulatory subunit 7 (MSS1) suppresses neuroinflammation. Plos One 7: e36142, 2012.

25. Bi W, Zhu L, Wang C, Liang Y, Liu J, Shi Q and Tao E: Rifampicin inhibits microglial inflammation and improves neuron survival against inflammation. Brain Res 1395: 12-20, 2011.

26. Xie Z, Smith CJ and Van Eldik LJ: Activated glia induce neuron death via MAP kinase signaling pathways involving JNK and p38. Glia 45: 170-179, 2004.

27. Bhat NR, Zhang P, Lee JC and Hogan EL: Extracellular signal-regulated kinase and p38 subgroups of mitogen-activated protein kinases regulate inducible nitric oxide synthase and tumor necrosis factor- $\alpha$ gene expression in endotoxin-stimulated primary glial cultures. J Neurosci 18: 1633-1641, 1998.

28. Madrid LV, Wang CY, Guttridge DC, Schottelius AJ, Baldwin AS Jr and Mayo MW: Akt suppresses apoptosis by stimulating the transactivation potential of the Rel A/p65 subunit of NF-кB. Mol Cell Biol 20: 1626-1638, 2000.

29. Downward J: Lipid-regulated kinases: some common themes at last. Science 279: 673-674,1998.

30. Li Y, Liu L, Barger SW, Mrak RE and Griffin WS: Vitamin E suppression of microglial activation is neuroprotective. J Neurosci Res 15: 163-170, 2001.

31. Brown GC and Neher JJ: Inflammatory neurodegeneration and mechanisms of microglial killing of neurons. Mol Neurobiol 41: 242-247, 2010.

32. Bi W, Zhu L, Jing X, Liang Y and Tao E: Rifampicin and Parkinson's disease. Neurol Sci 34: 137-141, 2013. 